\title{
Effect of doping on the radiation response of conductive $\mathrm{Nb}_{-} \mathrm{SrTiO}_{3}$ *
}

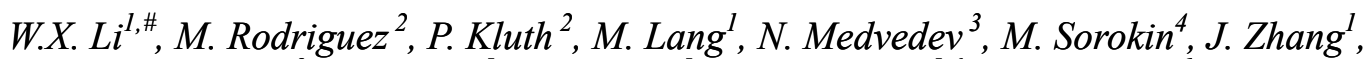 \\ B. Afra ${ }^{2}$, M. Bender ${ }^{5}$, D. Severin ${ }^{5}$, C. Trautmann ${ }^{5,6}$ and R. Ewing ${ }^{1}$ \\ ${ }^{1}$ University of Michigan, Ann Arbor, U.S.A.; ${ }^{2}$ The Australian National University, Australia; ${ }^{3}$ DESY, Germany; \\ ${ }^{4}$ Kurchatov Institute, Moscow, Russia; ${ }^{5}$ GSI, Darmstadt, Germany; ${ }^{6}$ TU Darmstadt, Germany
}

Based on the Coulomb-spike model, track formation is expected to depend on the electrical resistivity of a given material. Here, we report the first systematic study on ion tracks in doped $\mathrm{SrTiO}_{3}$ (STO) [1]. With the addition of low concentrations of $\mathrm{Nb}$ the resistivity of STO dramatically decreases covering the entire electronic regime from an insulating to conducting material.

Tracks were produced by exposing Nb-doped STO single crystals (thickness $\sim 40 \mu \mathrm{m}$ ) to $1.7-\mathrm{GeV} \mathrm{Au}$ or 2.0 $\mathrm{GeV} \mathrm{U}$ ions (see Table 1 for details) at the UNILAC accelerator. The $\mathrm{Nb}$ concentrations varied from $0,0.1$, and $1 \mathrm{wt} \%$ with corresponding resistivities of $\sim 10^{4}, 8 \times 10^{-2}$, and $3.5 \times 10^{-3} \Omega \mathrm{cm}$, respectively. The irradiated samples were investigated using transmission small-angle $\mathrm{x}$-ray scattering (SAXS) at the Australian Synchrotron in Melbourne. Crushed samples were inspected by transmission electron microscopy (TEM).

Tracks are observed by TEM in both undoped and $\mathrm{Nb}$ doped STO samples, despite the significant decrease in resistivity (Fig. 1). The tracks are parallel aligned and evident by the dark contrast of their damage trails with respect to the unirradiated matrix. In comparison to the sharp contrast between the track core and boundary in apatite [2], the contrast of tracks in undoped and $\mathrm{Nb}$ doped STO is rather weak. Independent of the $\mathrm{Nb}$-doping level, the track radius deduced from TEM images is rather similar ( 20-30 $\AA)$ for all samples. However, the weak contrast prevents a precise determination of the track radii by TEM. Track radii were also deduced from SAXS measurements by fitting the intensities data of the tracks as a function of the scattering vector. The SAXS track radii are consistent with the values as observed by TEM (Table 1). In contrast to the limited number of tracks in a localized area as observed by TEM, the strong scattering oscillations from a very large number of well aligned, identical tracks in a bulk sample, as detected by SAXS, provide a very reliable means for determining the mean track radius. The SAXS measurements further confirm that the $\mathrm{Nb}$-doping has no evident influence on the track size in STO.

There are two different possibilities that explain the independence of track formation on the $\mathrm{Nb}$-doping level. Firstly, although the density of the conduction electrons significantly increases up to $\sim 10^{20} \mathrm{~cm}^{-3}$ upon doping, this number is still too low to influence the cooling of the electronic subsystem as compared with the number of excited electrons $\left(\sim 10^{22} \mathrm{~cm}^{-3}\right)$ in the track core. Secondly,

* Work supported by the Office of Basic Energy Sciences of the U.S. DOE (DE-FG02-97ER45656).

"wxli@umich.edu as implied by the non-thermal model, the difference in the type of bonding between metals and dielectrics may be important for track formation because ionization might not so much change metallic bonds, while the covalent or ionic bonds can be broken. However, the low concentration doping in this study does not change the bonding type, just the electronic conductivity, and therefore has probably no significant effect on track formation.
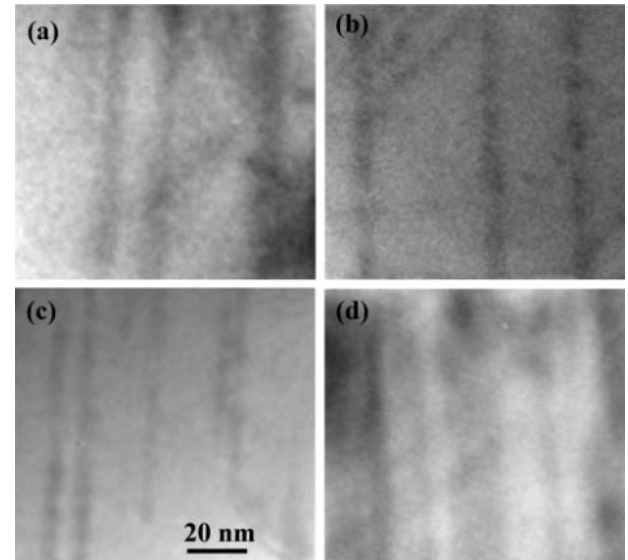

Figure 1: TEM images showing the morphologies of tracks created by $2.0-\mathrm{GeV} \mathrm{U}$ ions at room temperature in (a) undoped STO and (b) $1 \mathrm{wt} \% \mathrm{Nb}$-doped STO, and by 1.7-GeV Au ions at $24 \mathrm{~K}$ in (c) undoped STO and (d) 1 wt $\% \mathrm{Nb}$-doped STO.

Table 1: Irradiation parameters and track radii deduced from SAXS measurements.

\begin{tabular}{|c|c|c|c|}
\hline $\begin{array}{c}\text { Doping Nb } \\
(\mathrm{wt} \%)\end{array}$ & $\begin{array}{c}\text { Irradiation } \\
\text { temperature }\end{array}$ & $\begin{array}{c}\text { Ions } \\
(\mathrm{GeV})\end{array}$ & $R(\AA)$ \\
\hline 0 & $\mathrm{RT}$ & $\mathrm{U}(2.0)$ & $31.3(0.1)$ \\
\hline 0.1 & $\mathrm{RT}$ & $\mathrm{U}(2.0)$ & $30.7(0.2)$ \\
\hline 1 & $\mathrm{RT}$ & $\mathrm{U}(2.0)$ & $31.2(0.2)$ \\
\hline 0 & $\mathrm{RT}$ & $\mathrm{Au}(1.7)$ & $22.8(0.5)$ \\
\hline 0.1 & $\mathrm{RT}$ & $\mathrm{Au}(1.7)$ & $23.6(0.5)$ \\
\hline 1 & $\mathrm{RT}$ & $\mathrm{Au}(1.7)$ & N.A. \\
\hline 0 & $24 \mathrm{~K}$ & $\mathrm{Au}(1.7)$ & $22.8(0.2)$ \\
\hline 0.1 & $24 \mathrm{~K}$ & $\mathrm{Au} \mathrm{(1.7)}$ & $19.8(0.3)$ \\
\hline 1 & $24 \mathrm{~K}$ & $\mathrm{Au}(1.7)$ & N.A. \\
\hline
\end{tabular}

[1] W.X. Li et al., accepted in Nucl. Instr. Meth. (2013).

[2] W.X. Li, L.M. Wang, M. Lang, C. Trautmann, and R. Ewing, Earth Planet. Sci. Lett. 302 (2011) 227. 